\title{
Particle mixing and separation in a binary solids floating fluidized bed
}

\author{
G. Kwant ${ }^{\text {a }}$,W. Prins ${ }^{\text {b }}$, W.P.M. van Swaaij ${ }^{\text {b }}$ \\ "Gist-Brocades NV, PO Box 1, 2600 MA Delft, The Netherlands \\ b Twente University of Technology, PO Box 217, 7500 AE Enschede, The Netherlands
}

Received 5 January 1994; revised 31 October 1994

\begin{abstract}
The floating gas-solid fluidized bed (FGSFB) is a large-particle fluidized bed confined in a tapered column with inserted grids. By application of two types of particles in an FGSFB, a wide variety of operations can be carried out. The visual appearance and the hydrodynamic behaviour of such binary FGSFBs is reported in this paper. Two extreme types of behaviour were observed in the experimental set-up: (1) almost complete separation occurs in beds of two types of particle with the same diameter but a particle density ratio $\rho_{\mathrm{p}, 2} / \rho_{\mathrm{p}, 1}$ of ca. $2\left( \pm 1400 \mathrm{vs} \pm 700 \mathrm{~kg} / \mathrm{m}^{3}\right)$; (2) a relatively well-mixed situation is found in beds with two types of particle having the same density but a particle diameter ratio $d_{p, 2} d_{p, 1}$ of ca. 2 (3.0 vs 1.7 $\mathrm{mm}$ ). Experimentally determined pressure gradient values were always within the range of those measured for FGSFBs of the single-particle type. A measure for the separating force, the so-called separation tendency $W$, is derived and shown to be quantified by single-particle properties. Its value can be used to predict whether complete or intermediate mixing occurs, or if the binary FGSFB will be completely separated.
\end{abstract}

Keywords: Fluidized bed; Binary particles; Tapered column; Porosity distribution; Mixing; Segregation

\section{Introduction}

The floating gas-solids fluidized bed (FGSFB) [1,2] is a new development in gas-solids contactors. Particles are fluidized by a gas in a tapered column in which grid inserts ensure a certain degree of bed stabilization. At the bottom of the column, a gas velocity higher than the single-particle terminal velocity is required because there is no gas-distributing bottom plate. The superficial gas velocity decreases towards the top of the bed but always remains higher than the minimum fluidization velocity.

The FGSFB offers the possibility of operation with an inventory of two or more types of particle. A wide variety of applications can be carried out if different, for instance two, types of particle are used. One may apply:

- different types of catalyst material,

- a catalyst in combination with either a product adsorbent or a reactant desorbent, - a well-mixed bed or two completely separated beds,

\footnotetext{
* Corresponding author.
}

- continuous replacement of either one or both types of particle,

- continuous trickling down (up) of a heavier (lighter) particle type through a stationary bed,

- one particle type as a heat carrier.

A combination of various items in this list is possible and sometimes even required for continuous processes, e.g. continuous product adsorption can only be realized if particle replacement also takes place. The reader may note that the classifying property of the tapered FGSFB can be combined beneficially with the different (physico-)chemical characteristics (e.g. catalytic activity, adsorptive capacity) of the types of particles used in most of the above-mentioned cases.

A propitious application of two catalyst materials in an FGSFB column is illustrated by the following example. The FGSFB is a possible alternative for the parallel passage systems, applied at present to carry out the selective catalytic reduction (SCR) of $\mathrm{NO}_{x}$ by ammonia in flue gas from coal-fired boilers. In the SCR process, a high $\mathrm{NO}_{x}$ reduction is preferred while only a very small $\mathrm{NH}_{3}$-slip $(<5 \mathrm{vppm})$ is allowed to avoid condensation of ammonia sulfates in cool 


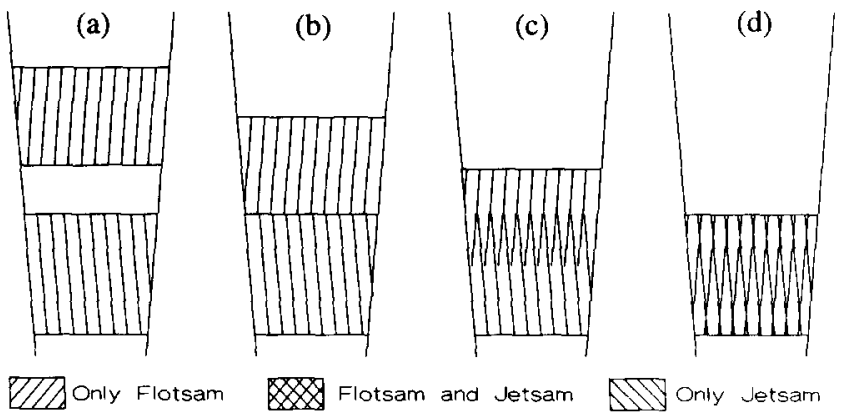

Fig. 1. Illustration of various appearances of a binary FGSFB: (a) two FGSFBs; (b) complete separation; (c) intermediate mixing; (d) completely mixed. Flotsam: lighter particle type; jetsam: denser particle type.

$(T<473 \mathrm{~K})$ sections of the gas treatment process. Ammonia slip always occurs due to imperfect contact of the flue gas with the catalyst. Therefore, generally a substoichiometric ammonia addition is applied to prevent surpassing of the $\mathrm{NH}_{3}$ concentration limit [3]. Consequently, the degree of $\mathrm{NO}_{x}$ reduction is limited to approximately $80 \%$. The residual nitrogen oxides concentration is $100-300 \mathrm{vppm}$, depending on the boiler type and on the fuel applied.

The use of higher ammonia levels (e.g. stoichiometric) can yield improved degrees of $\mathrm{NO}_{x}$ reduction (up to $100 \%$ ). Control of the outlet $\mathrm{NH}_{3}$ level can be accomplished by, for example, catalytic oxidation of unconverted ammonia to molecular nitrogen and water [4]. An FGSFB consisting of two, completely separated, catalyst beds can be applied [Fig. 1(a) and (b)] in such a case.

An important factor in the design of a binary FGSFB is the axial particle distribution and, accordingly, the degree of mixing of the different particle types. Consequently, it is useful to develop a method for prediction of the degree of particle separation in a binary FGSFB based on the column geometry and the particle distribution in single-particle beds only. Therefore, the hydrodynamic behaviour of FGSFBs with an inventory of two types of particle has been examined both theoretically and experimentally. From a theoretical analysis, a separation factor $(W)$ is derived here, which is solely determined by particle and fluid properties and by the local gas velocity employed. Experimental results are reported which confirm that $W$ can be applied quantitatively to indicate the extent of separation in an FGSFB. A design procedure is subsequently proposed in which this separation factor is applied to distinguish between a completely separated or completely mixed system.

\section{Background}

In the next paragraphs some of the work regarding mixing and separation in classical and tapered fluidized beds is surveyed. The literature examination is based mainly on the reviews presented by Rowe and Nienow [5] and Nienow and Chiba [6]. Furthermore, some literature on sedimentation of particles in a liquid will be discussed.

To denote the demixing tendency of solids in completely fluidized beds, we prefer to use the word 'separation' rather than 'segregation'. The latter term is applied here only if defluidization is meant.

\subsection{Mixing patterns}

It is well known that the mixing degree of solids in a binary fluidized bed with a constant cross-section is always between two extremes, i.e. complete separation and complete mixing of the solid phases [Fig. 1(a) to (d)] [5-7]. Completely separated binary beds consist of two distinct fluidized layers or beds, each containing one type of particle only [Fig. 1(a) and (b)]. Conversely, particles of each type are distributed uniformly over the entire solid-phase volume in well-mixed beds [Fig. $1(d)]$.

\subsection{Factors influencing mixing patterns}

The hydrodynamic behaviour of binary fluidized beds is strongly influenced by the differences in properties of the respective particles, the availability of internals and the prevailing fluidization regime.

Differences in particle properties in both fluidization $[5]$ and sedimentation [8,9] mainly affect the separation tendency. If these differences are relatively large, there is a correspondingly strong separation tendency which can eventually cause a binary fluidized bed to separate completely. It has been found that the ratio of particle densities $\left(\rho_{\mathrm{p}, 2} / \rho_{\mathrm{p}, 1}\right)$ has a stronger impact on separation tendency than the diameter ratio $\left(d_{\mathrm{p}, 2} / d_{\mathrm{p}, 1}\right)$. This is also reflected in Rowe and Nienow's terminology on particle identification: they indicate the particle type with the largest density as jetsam, almost irrespective of the diameter. However, if the densities are comparable the smallest particles are called flotsam [5]. Of course, the particles should be able to move for the separation tendency to be effective. This has been clarified by investigation of the influence of stirring on the solids mixing/separating behaviour at gas velocities around the minimum fluidization velocity but below the bubbling point [10]. It was found that separation is enhanced by gentle stirring. Later reports with the same apparatus [11] also mention a notable degree of demixing, even for small density differences.

Internals (grids, baffles, packing) have been shown to affect the hydrodynamic behaviour of fluidized beds by suppressing the formation and movement of heterogeneities [12-15]. It is commonly accepted that these heterogeneities are the main cause for solids mixing 
in bubbling fluidized beds [16] and therefore the mixing tendency in binary fluidized beds is suppressed by internals.

The fluidization regime which prevails also plays an important role in particle mixing processes [17]: vigourous particle movement yields a better-mixed bed. In the case of a binary bed, it may be impossible to indicate the fluidization regime for the entire bed: each particle type may be fluidized according to a different regime. For binary fluidized systems with a strong segregation tendency, it is reported that complete solids mixing is very difficult to achieve [5]. In its most extreme form $[12,14,18]$, a fluidized bed of fine particles is confined in a large-particle packed bed. One can also imagine that at a relatively high gas velocity the flotsam particles are subject to conditions normally encountered with turbulent fluidization, while the jetsam are fluidized in the bubbling regime.

\subsection{Quantification of mixing}

In homogeneously (liquid) fluidized beds, particle movement is random and therefore the mixing tendency can be described by a diffusion equation. This type of mixing is called micromixing [19]. Particle dispersion coefficients have been reported to be in the order of $10^{-3} \mathrm{~m}^{2} / \mathrm{s}$ for liquid-solid fluidized beds [20] and in the range of $10^{-3}-10^{0} \mathrm{~m}^{2} / \mathrm{s}$ for gas-solid fluidized beds, with values increasing on enlarging the scale of operation [19].

It has been shown that for gas-fluidized beds in the bubbling regime particle mixing is mainly caused by heterogeneities [16]. Particles travel upward in the wake of the moving voids while a downward movement is observed for particles in the bulk suspension. This type of (macro)mixing is, however, much more difficult to describe than micromixing because it requires detailed quantification of bubble wake movement and suspension flow. These phenomena are often very dependent on particle properties and on geometric factors such as the design of the gas distributor, the type of grids or internals employed and on column dimensions.

\subsection{Buoyancy forces in sedimentation}

Methods to quantify the size of separating forces in binary fluidized beds have been developed mainly for the more or less comparable process of sedimentation of binary solids material in liquids $[9,21-26]$. The formulated models differ in the quantification of the particle-particle interactions. Lockett and Al-Habbooby [9] applied the total fluid concentration ('porosity') in the Richardson and Zaki equation [8]:
$\frac{U}{U_{\mathrm{t}}}=\frac{U}{\left(\frac{\left(\rho_{\mathrm{p}}-\rho_{\mathrm{f}}\right) g V_{\mathrm{p}}}{C_{\mathrm{d}} A_{\perp} \frac{1}{2} \rho_{\mathrm{f}}}\right)}=\epsilon^{n}$

For the calculation of the slip velocity between individual particles and the fluid. They calculated the buoyancy force acting on a single particle on the basis of the fluid density. Values of the buoyancy force obtained from experimental falling velocities of particles in a fluid compared well with those derived from the model computations. Masliyah [21], however, derived that the slip velocity is a function of the suspension density:

$\rho_{\mathrm{sus}}=\epsilon \rho_{\mathrm{f}}+(1-\epsilon) \rho_{\mathrm{p}}$

rather than that of the fluid, and applied this result in the Richardson and Zaki equation [8]. Accordingly, in Eq. (1) the density difference must have a different value:

$\rho_{\mathrm{p}}-\rho_{\mathrm{f}} \Rightarrow \rho_{\mathrm{p}}-\rho_{\text {sus }}$

At almost the same time, Mirza and Richardson [22] reported that the experimental slip velocity compares well with that calculated by applying the Richardson and Zaki equation [8]. The power $n$ in Eq. (1), however, was higher $(+0.4)$ than that for single-particle systems, which was explained by the different particle-particle interaction in a binary system. The authors used the fluid density to describe the buoyancy action. Juma and Richardson [23] also applied the fluid density rather than the suspension density to account for buoyancy in a description of mixing and separation in a fluidized bed. Patwardhan and Tien [24] modified the result obtained for sedimentation by Masliyah [21]. A cell model was applied (each cell containing a single particle and a certain amount of fluid), assuming that every single cell in a binary mixture occupies the same volume. The fluid concentration thus obtained is different for cells containing unlike particle types. These new porosity values are subsequently applied in the relation for the slip velocity presented earlier by Masliyah [21]. Law et al. [25] showed that the models of Masliyah [21] and Patwardhan and Tien [24] predict the settling velocities better than the models of Lockett and AlHabbooby [9] and Mirza and Richardson [22]. On comparing experimental results with predictions by a variety of models, Al-Naafa and Selim [26] concluded that slip velocities are best described by the so-called Selim model reported in 1983 [27,28]. Essentially, their model accounts for particle-particle interactions by applying the terminal falling velocity of the fastest falling particle, which is based on the suspension density [Eq. (3)]. The particle density applied in Eq. (3) is that of the slower falling particles.

This summary shows that, although progress has been made in describing the settling velocity of every con- 
stituent in a sedimenting binary mixture, discussion on the fundamental processes taking place is still not finished.

\subsection{Buoyancy in fluidized beds}

The definition of the net gravity force on a single particle in fluidized systems is, just like in sedimentation, sometimes also based on the difference between particle and suspension densities [29-31]. Arguments have been raised against this approach: a particle is surrounded by 'clear' fluid and therefore the buoyancy should be based on the fluid density. Some hydrodynamic models are indeed based on the fluid density instead of the suspension density $[8,32]$. In the theoretical part of this work the concept of buoyancy forces based on the suspension density is critically analyzed and the result is used for a clear definition of the separation tendency of a binary mixture of particles.

\subsection{Separation in tapered fluidized beds}

Experiments concerning liquid fluidization of a binary solids mixture in tapered columns have been reported only by Maruyama et al. [7]. They demonstrated the possibility of different degrees of mixing or separation in tapered binary fluidized beds. For gas-solids systems, only two studies are known to us in which the tapered bed is applied as a classifier for two types of particle $[33,34]$. However, detailed experimental information on particle distributions is lacking, and a theoretical model has not been developed for tapered fluidized beds of two or more particle types. In gas-solid tapered fluidized beds of a single particle type, the mixing of solids was observed [35-37] to be less than in classical fluidized beds at the same gas velocity. This is attributed to the suppression of void formation. However, at gas velocities over \pm 1.5 times the minimum fluidization velocity, no difference from cylindrical fluidized beds could be observed anymore.

\section{Previous observations in FGSFBs of a single particle type}

For a better understanding of the results reported in this paper, it is useful to review shortly the earlier observations for an FGSFB of a single particle type [2]. Fig. 2 shows a sketch of the top section of an FGSFB; it will be used hereafter for the description of the observed particle motion. The letters between brackets in the text refer to the letters used in Fig. 2. In the top of the FGSFB, dense layers with upwardmoving voids (A), a meandering 'plume' $(B)$, grids $(C)$ and a volume with solids downflow near the wall (D) can be distinguished. Visual observations revealed that

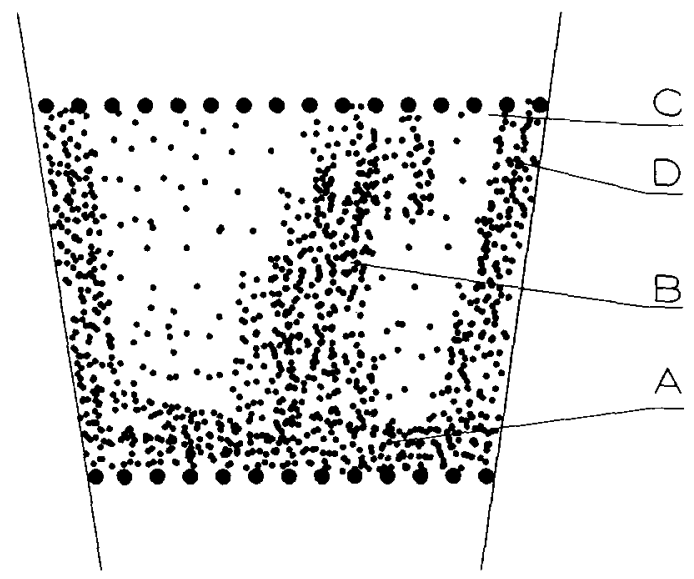

Fig. 2. FGSFB top section; (A) Heterogeneously fluidized layer on grid; (B) meandering 'plume' with particle upflow; (C) grid; (D) downflow current of particles.

particles move upward in plume (B) while trickling downward along the wall (D).

The processes which contribute to solids mixing in a cylindrical fluidized bed are mainly bubble or void movements and gross circulation patterns. However, in the FGSFB, bubble or void movement was observed to occur only within the small layers just above a grid (A in Fig. 2). Therefore this mechanism seems to be relatively unimportant for overall solids mixing. On the other hand, due to these voids, particles are forced to move and therefore loosening of their packing occurs. Then separation forces can take effect and consequently cause a certain degree of separation. An important role in solids mixing and separation processes is played by the meandering 'plume' in the FGSFB (Fig. 2, B). It lifts particles to higher positions and consequently contributes to mixing. However, this gross circular motion is restrained by the available grids. Separation, due to differences in inertia forces, may also be enhanced because of the lateral acceleration. Quantification of the impact of grids and of the 'plume' on the solids mixing and separation behaviour of binary FGSFBs is very complicated and will not be discussed further.

\section{Theoretical}

The main purpose of this section is to derive a simple expression for the tendency of a binary fluidized bed towards solids separation using only information on single-particle fluidized beds.

\subsection{Separation model formulation}

The force balance on any single fluidized particle, in the absence of mixing forces, can be written as: 
$f_{\text {net }}=f_{\mathrm{fr}}+f_{\mathrm{b}}-f_{\mathrm{g}}=C_{\mathrm{d}}(\epsilon) \frac{A_{\mathrm{vp}}}{V} \frac{1}{2} \rho_{\mathrm{f}} U^{2}-g\left(\rho_{\mathrm{p}}-\rho_{\mathrm{f}}^{*}\right)$

in which $\rho_{\mathrm{f}}^{*}$ is the density of either the fluid or the suspension. The friction force $f_{\mathrm{fr}}$ on a single fluidized particle is determined by the superficial velocity and the friction coefficient $C_{\mathrm{d}}(\epsilon)$. A single voidage function is generally applied to account for the effect of particle-particle interactions on the value of the friction factor $C_{\mathrm{d}}(\epsilon)$. It is frequently obtained from the Richardson and Zaki equation [Eq. (1)] [8]:

$C_{\mathrm{d}}(\epsilon)=C_{\mathrm{d}}(\epsilon=1) F(\epsilon)=C_{\mathrm{d}}(\epsilon=1) \epsilon^{-4.7}$

For large-particle systems in FGSFBs, Kwant [2] found experimentally:

$F(\epsilon)=\frac{1+23.5(1-\epsilon)}{\epsilon^{2}}$

The buoyancy force $f_{\mathrm{b}}$ in Eq. (4), for single-particle type systems, is in principle based on either the fluid density $\left(\rho_{\mathrm{f}}^{*}=\rho_{\mathrm{f}}\right)$ or the suspension density $\left(\rho_{\mathrm{f}}^{*}=\rho_{\mathrm{sus}}\right)$. As mentioned above, there is no agreement in the literature on the exact definition which needs to be applied. Generally, the volume occupied by a fluidized particle is considered to be that of the single particle only. However, a particle moving in a single-particle type fluidized bed does not only displace its own ("physical') volume but also the surrounding fluid. In fact, it cannot exist in a fluidized state without the surrounding envelope of fluid. In the following, the particle volume is therefore considered together with the envelope of fluid required to satisfy the force balance, yielding the 'unit cell' volume, as illustrated by Fig. 3:

$V_{\mathrm{uc}, i}=\frac{\frac{\pi}{6} d_{\mathrm{p}, \mathrm{I}}^{3}}{1-\epsilon_{i}^{\mathrm{o}}}$

Such a formulation is frequently referred to as a 'cell model'. The currently applied model is an extension of that used by Patwardhan and Tien [24] for sedimentation of binary mixtures. They assumed that every

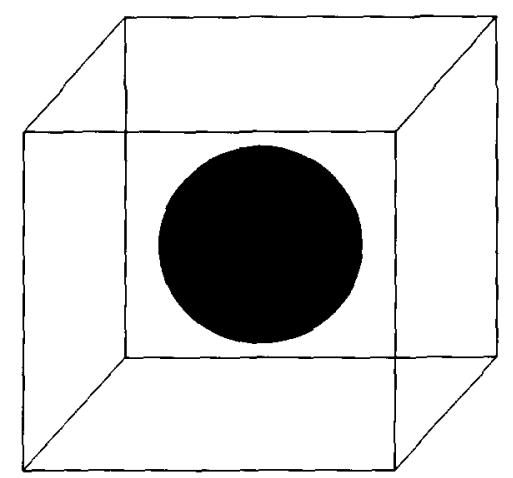

Fig. 3. Illustration of a unit cell with one particle. unit cell has the same volume, independent of the type of particle it contains. In the present model it is postulated that every unit cell possesses the same volume in a binary bed that it occupied in a single-particle type fluidized bed under the same physical circumstances.

This unit cell can also be projected in binary fluidized beds. The particle-particle interactions are assumed to be the same as those in a single-particle type fluidized bed. However, for a unit cell in a binary bed, it is possible to derive an expression for the buoyancy force which is based upon the 'suspension' density, now defined by:

$\rho_{\mathrm{sus}}=X_{1} \rho_{\mathrm{uc}, 1}+X_{2} \rho_{\mathrm{uc}, 2}$

where the volumetric solids fractions $X_{1}$ and $X_{2}$ follow from:

$X_{1}=\frac{N_{1} V_{\mathrm{uc}, 1}}{N_{1} V_{\mathrm{uc}, 1}+N_{2} V_{\mathrm{uc}, 2}}=1-X_{2}$

The reader should note that the extra particle-particle interactions for the binary bed are now described by the value of $X_{i}$ rather than by modifying the unit cell volume as in the model defined by Patwardhan and Tien [24]. Subsequently, the net force on the unit cells of type 1 and 2 follows from:

$$
\begin{gathered}
f_{\text {net, } 1}=\left(\rho_{\mathrm{uc}, 1}-\rho_{\mathrm{sus}}\right) g=\left(1-X_{1}\right)\left(\rho_{\mathrm{uc}, 1}-\rho_{\mathrm{uc}, 2}\right) \\
g=\left(1-X_{1}\right) g W=-f_{\mathrm{net}, 2}
\end{gathered}
$$

This is an important result since it states that in every binary system with $W$ different from 0 there is always a separating force if two types of particle are mixed, i.e. $0<X_{1}<1$. In other words, if a mixture of two particle types is fluidized, separation will occur for $W \neq 0$ and when mixing forces are absent. The magnitude of the driving force towards separation is strongly dependent on $W$. Therefore $W$ is a good measure for the separation tendency in binary beds and thus it will be referred to in the following.

Another important conclusion, complementary to the one above, is that separation occurs in those binary systems where mixing is absent and $W>0$. In fluidized systems there is always a certain degree of mixing (however defined or measured). The degree of mixing can now be estimated qualitatively, for example:

- homogeneous mixing in systems with high $W$ values indicates the occurrence of profound mixing forces; - complete separation in systems with small $W$ values indicates the almost complete absence of mixing forces.

Another way of qualitatively indicating the degree of mixing is by applying the local fraction of a unit cell type, e.g. $X_{1}$. Values of $X_{1}$ of both 1 in the top layer of the bed and zero below it indicate a completely separated situation, while values equal to: 
$X_{1, \text { ext }}=\frac{N_{1} V_{\mathrm{p}, 1}}{N_{1} V_{\mathrm{p}, 1}+N_{2} V_{\mathrm{p}, 2}}$

can only be obtained in an ideally mixed bed. Measurement of $X_{1}$ can be accomplished by using experimental axial pressure drop profiles. The local pressure drop due to the presence of a mixture of two types of particle over a differential height $\mathrm{d} H$ can be written as:

$A_{\perp} \mathrm{d} P=\left(\rho_{\mathrm{p}, 1}-\rho_{\mathrm{f}}\right) g \mathrm{~d} V_{\mathrm{p}, 1}+\left(\rho_{\mathrm{p}, 2}-\rho_{\mathrm{f}}\right) g \mathrm{~d} V_{\mathrm{p}, 2}$

Over a differential height $\mathrm{d} H$, the porosity and the volume fraction $X_{i}$ are effectively constant, by which one obtains:

$\mathrm{d} V_{\mathrm{p}, i}=X_{i}\left(1-\epsilon_{i}^{\mathrm{o}}\right) \mathrm{d} V_{\mathrm{tot}}=X_{i}\left(1-\epsilon_{i}^{\mathrm{o}}\right) A_{\perp} \mathrm{d} H$

Together with the pressure drop due to particle-gas friction in a single-component bed, described by:

$\mathrm{dP}_{i}^{\circ}=\left(1-\epsilon_{i}^{\circ}\right)\left(\rho_{\mathrm{p}, i}-\rho_{i}\right) g \mathrm{~d} H$

it is possible to rewrite Eq. (12) to give:

$\frac{\mathrm{d} P}{\mathrm{~d} H}=X_{1} \frac{\mathrm{d} P_{1}^{\circ}}{\mathrm{d} H}+X_{2} \frac{\mathrm{d} P_{2}^{\circ}}{\mathrm{d} H}$

By rearranging Eq. (15) and using Fq. (9) to eliminate $X_{2}$, one obtains for a binary bed:

$X_{1}=\frac{\frac{\mathrm{d} P}{\mathrm{~d} H}-\frac{\mathrm{d} P_{2}^{\circ}}{\mathrm{d} H}}{\frac{\mathrm{d} P_{1}^{\circ}}{\mathrm{d} H}-\frac{\mathrm{d} P_{2}^{\circ}}{\mathrm{d} H}}$

Quantifying $W$ for binary fluidized beds is now possible if the above equations are applied together with correlations for the porosity-velocity relation in singleparticle type fluidized beds [e.g. Eq. (6)]. It should be noted that the local porosity in an FGSFB is dependent on the type of particle, through $U_{\mathrm{t}, i}$, and on the fluid flowrate, the axial position in the column and the column geometry, through $U$ :

$U=\frac{\Phi_{v}}{\frac{\pi}{4}[\mathrm{D}+\tan (\alpha) H]^{2}}$

$W$ can be easily rewritten in terms of pressure drop in single-particle type fluidized beds because:

$$
\begin{aligned}
W=\rho_{\mathrm{uc}, 1}- & \rho_{\mathrm{uc}, 2}=\left(\rho_{\mathrm{uc}, 1}-\rho_{\mathrm{f}}\right)-\left(\rho_{\mathrm{uc}, 2}-\rho_{\mathrm{f}}\right) \\
= & \left(1-\epsilon_{1}^{\mathrm{o}}\right)\left(\rho_{\mathrm{p}, 1}-\rho_{\mathrm{f}}\right)-\left(1-\epsilon_{2}^{\mathrm{o}}\right)\left(\rho_{\mathrm{p}, 2}-\rho_{\mathrm{f}}\right)
\end{aligned}
$$

It then follows for $W$, by introduction of Eq. (14):

$W=\frac{1}{g} \frac{\mathrm{d} P_{1}^{\mathrm{o}}}{\mathrm{d} H}-\frac{1}{g} \frac{\mathrm{d} P_{2}^{\circ}}{\mathrm{d} H}$

This result renders it possible to calculate $W$ using Eqs. (4), (5), (14) and (19). For the local value of $W$ in a fluidized binary system in a tapered column, Eq. (16) should be applied to calculate the local gas velocity $U$, required in Eq. (4), while Eq. (6) should be used for quantification of $F(\epsilon)$ in Eqs. (4) and (5).

\subsection{Model calculation}

It is interesting to illustrate the influence of the particle diameter ratio, of the particle density ratio and of the superficial gas velocity on the separation tendency $W$. In Fig. 4 the values of $W$ calculated from Eqs. (4)-(6), Eqs. (17) and (19) are presented as a function of the ratio of the particle densities (top horizontal axis) and the particle diameters (bottom horizontal axis). This plot shows clearly that particle diameter ratios have a smaller effect on $W$ than comparable density ratios. Such a finding is in accordance with observed experimental results reported in the literature $[5,6]$.

\subsection{Regime quantification}

Because $W$ is defined as the separation tendency, its value will be compared to the experimentally observed separation behaviour. Two extreme values can be obtained: a certain high one, above which a bed is completely separated, and a low value below which complete mixing occurs. It should be noted that both boundary values are dependent on the mixing action in the fluidized bed: the stronger this is, the higher these

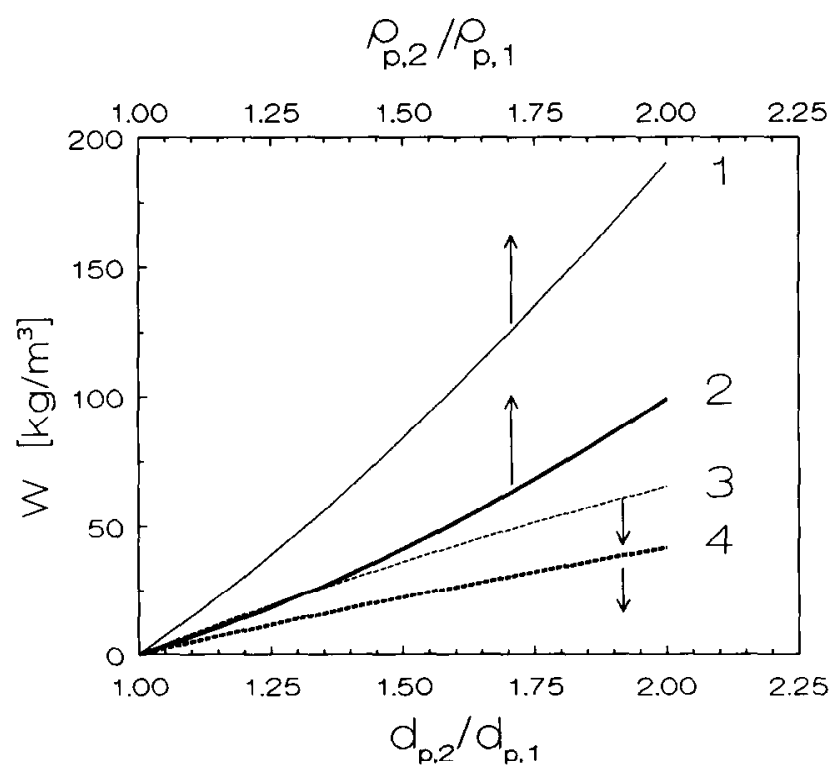

Fig. 4. Calculated separation tendency $W$ vs. density ratio $\rho_{\mathrm{p}, 2} \rho_{\mathrm{p}, 1}$ (top horizontal axis, 1 and 2) and diameter ratio $d_{p, 2} / d_{p, 1}$ (bottom horizontal axis, curves 3 and 4) shown are calculated values for two local gas velocities: $0.5 U_{t, 1}$ for curves 1 and $3,0.75 U_{t, 1}$ for curves 2 and 4 . Fixed values are used for properties of the smallest (flotsam) particles, i.e. $d_{\mathrm{p}, \mathrm{t}}=0.0017 \mathrm{~m}$ and $\rho_{\mathrm{p}, \mathrm{t}}=677 \mathrm{~kg} / \mathrm{m}^{3}$. The direction of the arrows indicates the corresponding horizontal axis. 
values are. Therefore the exact values will be dependent on factors such as the geometry of the experimental column, the fluidization regime and the presence and shape of internals. In fact, one can quantify and compare the mixing action in different fluidized systems by the boundary values of $W$. Further quantification may be the subject for future research.

\section{Experimental}

Experiments have been carried out in a squared tapered column described in detail elsewhere [1,2]. This column, constructed of transparent acrylic plates, is shown schematically in Fig. 5 . It has a length of $2 \mathrm{~m}$, an entrance edge of $0.1 \mathrm{~m}$ and an apex angle of $4.7^{\circ}$. Ambient air can be passed through the column at flowrates from $200-1000 \mathrm{~m}^{3} / \mathrm{h}$. Between two successive grids there is a volume of $0.0025 \mathrm{~m}^{3}$. The grid, also shown in Fig. 5, consists of $5 \mathrm{~mm}$ diameter cylindrical rods separated by a distance of $10 \mathrm{~mm}$. Properties of the particles applied are presented in Table 1 while

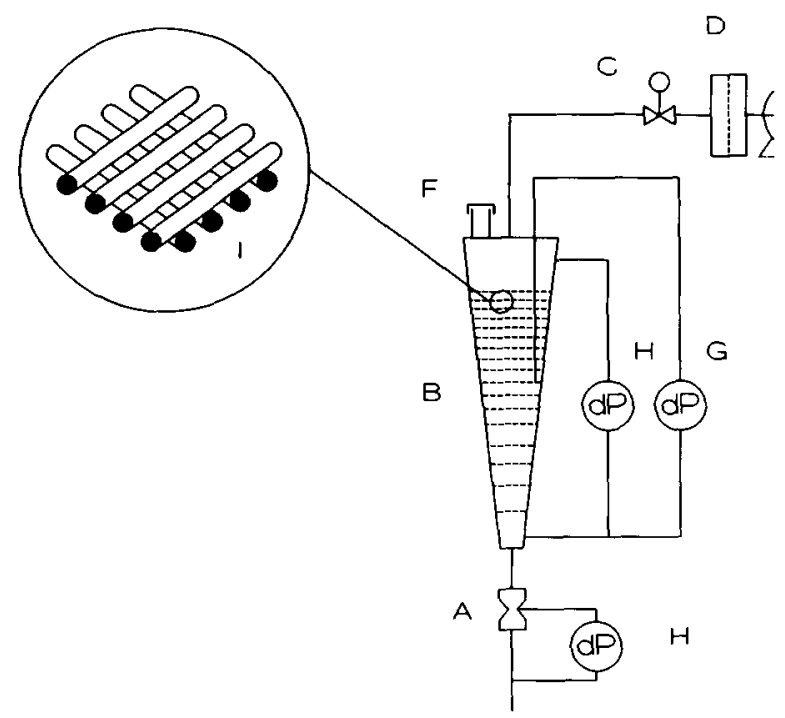

Fig. 5. Set-up of the FGSFB for ambient conditions. A: Venturi tube; B: tapered column; C: valve; D: filter; E: ventilator; F: solids feed point; G: pressure probe; H: pressure tap; I: grid illustration.

Table 1

Properties of spherical particles used in this work

\begin{tabular}{lclrr}
\hline Type & $\begin{array}{l}\rho_{\mathrm{p}} \\
\left(\mathrm{kg} / \mathrm{m}^{3}\right)\end{array}$ & $\begin{array}{l}d_{\mathrm{p}} \\
(\mathrm{m})\end{array}$ & $\begin{array}{l}U_{\mathrm{t}} \\
(\mathrm{m} / \mathrm{s})\end{array}$ & \multicolumn{1}{c}{$\begin{array}{l}\mathrm{Re}_{\mathrm{t}} \\
(-)\end{array}$} \\
\hline Silica & 677 & 0.0017 & 4.95 & 673 \\
Silica & 727 & 0.0023 & 6.40 & 1177 \\
Silica & 744 & 0.0030 & 7.39 & 1776 \\
Polymer & 1465 & 0.0030 & 10.34 & 2490 \\
Glass & 2847 & 0.0008 & 6.37 & 407 \\
Glass & 2920 & 0.0011 & 7.78 & 622 \\
Glass & 2887 & 0.0013 & 9.59 & 997 \\
\hline
\end{tabular}

the combinations of particles used to create binary FGSFBs are shown in Table 2.

To start an experiment, a single-particle type FGSFB with air at ambient conditions is established. The bed mass of this type of particle is kept constant during the experiment. Then a specified amount of the second particle type is added intermittently. A pressure-drop profile is measured after every mass addition using a movable pressure probe which is positioned on the central column axis. During the experiments, visual observation was possible and the appearance is reported.

\section{Results}

\subsection{Visual observations}

Experiments have been carried out with various combinations of particles. Hereafter results are evaluated of observations made with binary FGSFBs consisting of combinations of particles with the largest difference in diameter, in density or in both (Table 2).

First the visual behaviour of binary FGSFBs with a large differences in particle density was investigated. To this end, a bed of relatively dense polymer particles (Table 1) was created with about half the number of grids (viz. the upper ones) present above the bed inventory. Silica particles of the same diameter $(0.0030$ $\mathrm{m})$ were then introduced from the top of the column in amounts of $0.2 \mathrm{~kg}$ (Table 2, experiment No. I). It was observed that silica particles hardly penetrated into the polymer bed; instead, they stayed floating on top of it. Only a few silica particles were mixed with the polymer particles in the intergrid volume at top of the initial polymer bed. Increasing the silica inventory did not change the visual bed appearance: the silica-rich part of the bed only increased in height.

The reversed filling procedure was also applied (Table 2, II): an FGSFB of $0.0030 \mathrm{~m}$ silica particles was created after which $0.0030 \mathrm{~m}$ polymer particles were added. These polymer particles descended through the silica bed and formed a new fluidized bed below the bottom of the initial silica bed. If a sufficient quantity of polymer is added, then a transfer of the silica-rich part to a higher position in the column occurred. The same observations were made if silica particles were used with a diameter of $0.0017 \mathrm{~m}$ instead of $0.0030 \mathrm{~m}$. Little effect on the mixing/separation behaviour seemed to occur using silica particles with a different diameter.

Subsequently, an experiment was carried out to investigate the influence of particle diameter while using particles of approximately the same density. An FGSFB of $0.0030 \mathrm{~m}$ silica particles was created, after which quantities of $0.2 \mathrm{~kg}$ of $0.0017 \mathrm{~m}$ silica particles were successively introduced (Table 2, IV). 
Table 2

Combinations of particles used in binary FGSFBs of which visual observations are reported

\begin{tabular}{|c|c|c|c|c|}
\hline $\begin{array}{l}\text { Experiment } \\
\text { number }\end{array}$ & $\begin{array}{l}\text { Particle } \\
\text { Type I }\end{array}$ & $\begin{array}{l}\text { Particle } \\
\text { Type II }\end{array}$ & Constant amount & Remarks \\
\hline I & $\begin{array}{l}\text { Polymer } \\
(0.0030 \mathrm{~m})\end{array}$ & $\begin{array}{l}\text { Silica } \\
(0.0030 \mathrm{~m})\end{array}$ & $\begin{array}{l}\text { Polymer } \\
(0.0030 \mathrm{~m})\end{array}$ & $\begin{array}{l}\text { Large density } \\
\text { difference }\end{array}$ \\
\hline II & $\begin{array}{l}\text { Polymer } \\
(0.0030 \mathrm{~m})\end{array}$ & $\begin{array}{l}\text { Silica } \\
(0.0030 \mathrm{~m})\end{array}$ & $\begin{array}{l}\text { Silica } \\
(0.0030 \mathrm{~m})\end{array}$ & $\begin{array}{l}\text { Large density } \\
\text { difference }\end{array}$ \\
\hline III & $\begin{array}{l}\text { Polymer } \\
(0.0030 \mathrm{~m})\end{array}$ & $\begin{array}{l}\text { Silica } \\
(0.0017 \mathrm{~m})\end{array}$ & $\begin{array}{l}\text { Silica } \\
(0.0017 \mathrm{~m})\end{array}$ & $\begin{array}{l}\text { Large density and } \\
\text { diameter differences }\end{array}$ \\
\hline IV & $\begin{array}{l}\text { Silica } \\
(0.0030 \mathrm{~m})\end{array}$ & $\begin{array}{l}\text { Silica } \\
(0.0017 \mathrm{~m})\end{array}$ & $\begin{array}{l}\text { Silica } \\
(0.0017 \mathrm{~m})\end{array}$ & $\begin{array}{l}\text { Large diameter } \\
\text { difference }\end{array}$ \\
\hline V & $\begin{array}{l}\text { Silica } \\
(0.0030 \mathrm{~m})\end{array}$ & $\begin{array}{l}\text { Silica } \\
(0.0017 \mathrm{~m})\end{array}$ & $\begin{array}{l}\text { Silica } \\
(0.0030 \mathrm{~m})\end{array}$ & $\begin{array}{l}\text { Large diameter } \\
\text { difference }\end{array}$ \\
\hline VI & $\begin{array}{l}\text { Silica } \\
(0.0030 \mathrm{~m})\end{array}$ & $\begin{array}{l}\text { Silica } \\
(0.0023 \mathrm{~m})\end{array}$ & $\begin{array}{l}\text { Silica } \\
(0.0023 \mathrm{~m})\end{array}$ & $\begin{array}{l}\text { Moderate diameter } \\
\text { difference }\end{array}$ \\
\hline VII & $\begin{array}{l}\text { Polymer } \\
(0.0030 \mathrm{~m})\end{array}$ & $\begin{array}{l}\text { Glass } \\
(0.0013 \mathrm{~m})\end{array}$ & $\begin{array}{l}\text { Polymer } \\
(0.0030 \mathrm{~m})\end{array}$ & $\begin{array}{l}\text { Large density and } \\
\text { diameter differences }\end{array}$ \\
\hline
\end{tabular}

Clearly, a different situation occurred compared to the situation in the afore-mentioned experiments, since both types of particle were found throughout the entire bed volume. Upon further increasing the amount of small particles, some demixing seemed to occur because these particles were increasingly found in the top part of the bed. Mixing was also observed if the reversed filling procedure was applied, i.e. when the larger (silica $0.0030 \mathrm{~m}$ ) particles were added to the bed inventory of $0.0017 \mathrm{~m}$ silica particles (Table 2, V). Now, however, it was found that increased homogeneity in the bed occurred upon augmenting the amount of $0.0030 \mathrm{~m}$ silica particles. Clearly, the $0.0030 \mathrm{~m}$ silica particles are preferentially present at the bottom of the bed but, upon their increasing in the bed inventory, these cannot entirely displace the smaller particles.

In the last experiment, polymer particles were introduced in the column together with the smaller but denser glass particles (Table 2, VII) with almost the same free-falling velocity $U_{\mathrm{t}}$ (Table 2 ). A well-mixed bed was observed showing hardly any tendency towards separation. This was a surprising result in view of the high density ratio (ca. 2) of both particle types: experiments with silica and polymer particles having (nearly) the same density ratio showed complete separation. An explanation may be the small value of $W$; this will be discussed in a following section.

\subsection{Pressure-drop measurements}

The effect of a large difference in particle density on the local axial pressure gradient is shown in Fig. 6 for beds of different inventories. It should be noted that the figure represents only that part of the total pressure drop which is caused by the friction between gas and particles. The pressure drop due to the friction of the gas with the column and grids, and also the pressure drop from the superficial gas velocity variation along the column, are subtracted from the measured value. For comparison, the profiles for a single particle bed are given as well; these were calculated from the expressions derived for the FGSFB, i.e. Eqs. (4)-(6), (14) and (17) in this work. The experimental pressure gradients for relatively high and low velocity were equal to those obtained from a single-particle type bed of the jetsam and flotsam particle type respectively. If the bed mass of polymer (jetsam) particles increases, the sudden variation in pressure gradient was found at lower gas velocity. This compares to positions higher in the column, which is in accordance with the visually observed behaviour.

In Fig. 7 , pressure-gradient profiles are presented for beds of particles with a difference in diameter rather than density. The scales of both axes in this figure are identical to those in Fig. 6. Upon close examination, one may note that the single-particle curves seem to be approached in the bottom and the top of the bed, especially for the binary system of $0.0023 \mathrm{~m}$ and $0.0030 \mathrm{~m}$ silica particles [Fig. (7a)]. Therefore, that bed can be regarded as only moderately mixed for small masses of $0.0030 \mathrm{~m}$ silica: the experimental curve tends to the single-particle curve. More complete mixing was obtained upon increasing the bed inventory of 0.0030 $\mathrm{m}$ silica in the system of $0.0023 \mathrm{~m}$ and $0.0030 \mathrm{~m}$ silica, because the curve of experimental points shows a nearly constant distance from the single-particle curves.

\section{Discussion}

In this section the results obtained are discussed, starting with the visual observations. Limiting values of $W$ are derived corresponding to the regimes of complete mixing, of complete separation and of the 

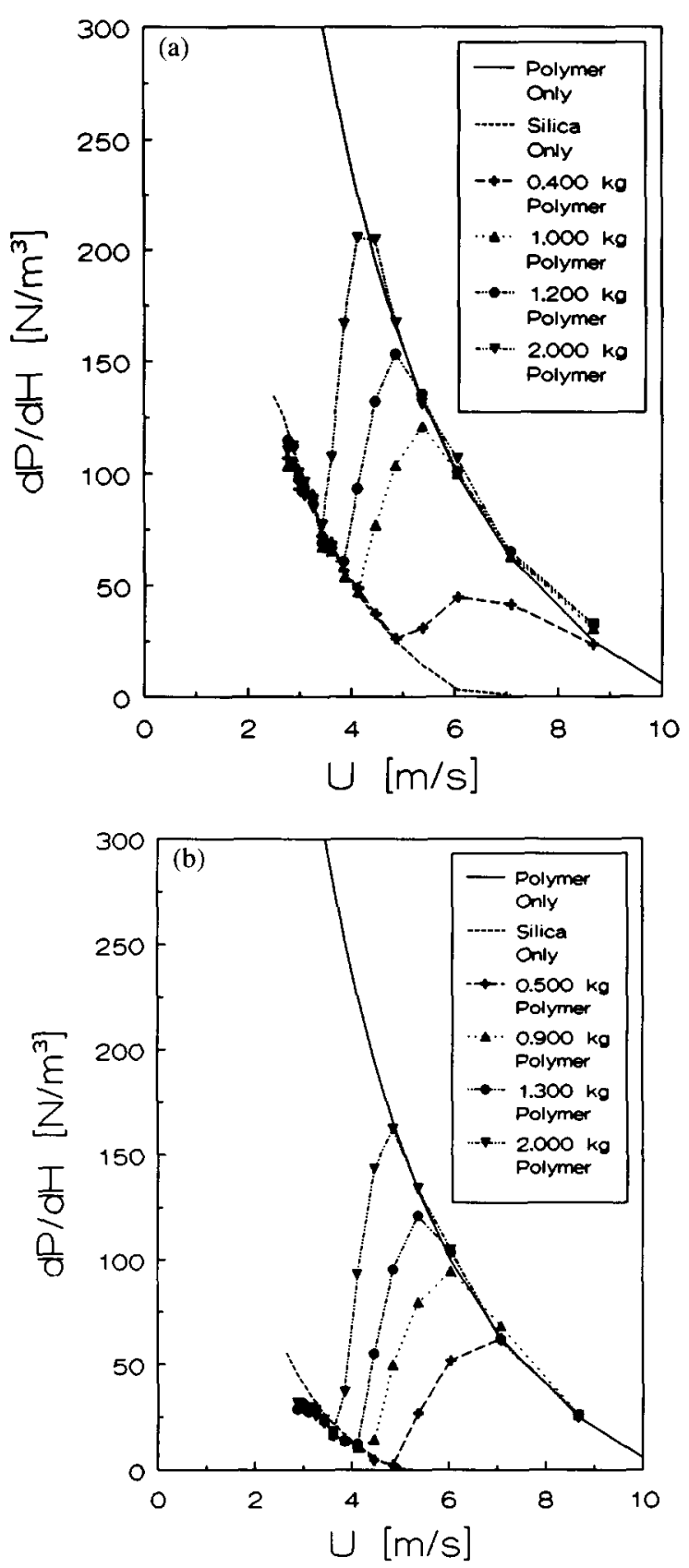

Fig. 6. Local pressure gradient due to gas-particle friction against the local superficial gas velocity. The latter is a function of the axial position in the column. Shown are data beds containing various masses of polymer and $0.5 \mathrm{~kg}$ silica $0.0017 \mathrm{~m}$ (a) and of different masses of polymer and $1.5 \mathrm{~kg}$ of silica $0.0030 \mathrm{~m} \mathrm{(b)}$. Results are typical for a large particle density difference.

intermediate region. Finally, a design procedure for binary FGSFBs is suggested briefly.

\subsection{Visual observations}

The experimental results presented demonstrate that binary FGSFBs with quite different mixing/separation performances can be created. A considerable density
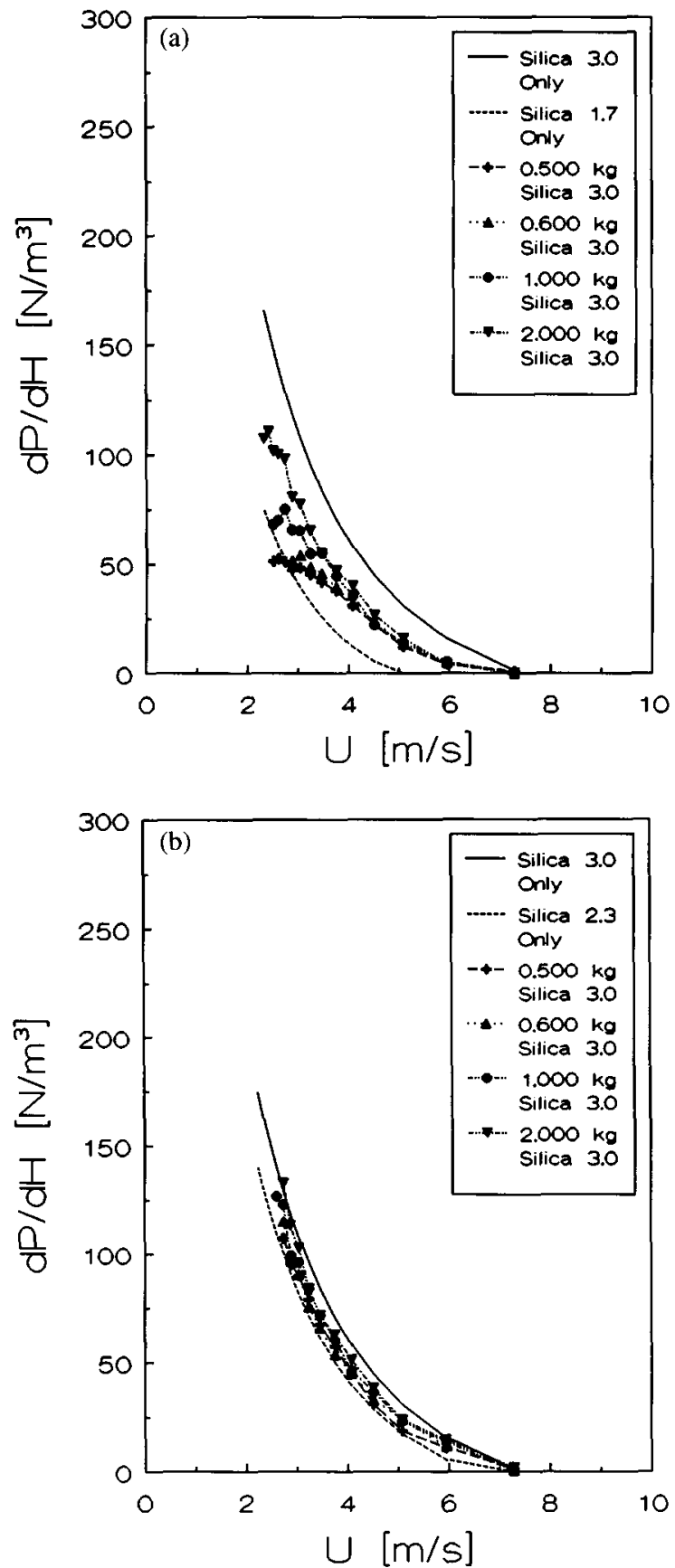

Fig. 7. Local pressure gradient due to gas-particle friction against the local superficial gas velocity. The latter is a function of the axial position in the column. Shown are data for beds with various masses of silica $0.0030 \mathrm{~m}$ and $0.8 \mathrm{~kg}$ silica $0.0017 \mathrm{~m} \mathrm{(a)} \mathrm{and} \mathrm{of} \mathrm{different}$ masses of silica $0.0030 \mathrm{~m}$ and $1.0 \mathrm{~kg}$ of silica $0.0023 \mathrm{~m}$ (b). Results are typical for a large particle diameter difference.

difference in the single-particle types of almost the same size led to completely separated beds. Consequently, the separation tendency in these beds was dominant over the mixing tendency. If two types of particle with a diameter ratio $d_{\mathrm{p}, 2} / d_{\mathrm{p}, 1}$ of almost 2 were introduced, keeping the densities of the individual particles approximately the same, mixing was substantial 
but not complete. Accordingly, the separation tendency of beds with a certain diameter ratio should be smaller than in beds with a comparable density ratio. This finding is in accordance with reported results [5] and the calculated results shown in Fig. 4.

In the glass-polymer system, complete separation was expected on the basis of the density ratio $\rho_{\mathrm{p}, 2} / \rho_{\mathrm{p}, 1}$ which was comparable to that of polymer-silica systems. Surprisingly, complete mixing occurred instead, as revealed by visual observation. Hereafter it will be shown that the separation tendency $W$ explains this behaviour.

\subsection{Pressure-drop measurement}

The behaviour reported above has been quantified using a pressure probe [2]. It was found that the local pressure drop, measured between two succeeding grids, was always approximately in the range of values of the single-particle type FGSFBs. This provides some support for the model assumption that each particle behaves as if it were available in a single-particle type bed.

In Figs. 8 and 9 the calculated values of the mixing parameter $X_{1}$ [Eq. (16)] are shown for a separated and a well-mixed system respectively. In the case of the separated system, the values are either zero or 1 denoting the presence of two beds of one single-particle type, the flotsam on top of the jetsam. In the completely mixed situation (Table 2, VI), the scatter in the data is increased. However, the mean values of $X_{1}$ do agree reasonably well with those expected from Eq. (11), which are shown in the figure by horizontal lines.

\subsection{Separation tendency $W$}

For every experiment, the value of the separation tendency $W$ is compared to the mixing situation. The reader should note that the product $W g$ follows from Figs. 6 and 7 as the vertical distance between the calculated single-particle curves [Eq. (7)]. Upon com-

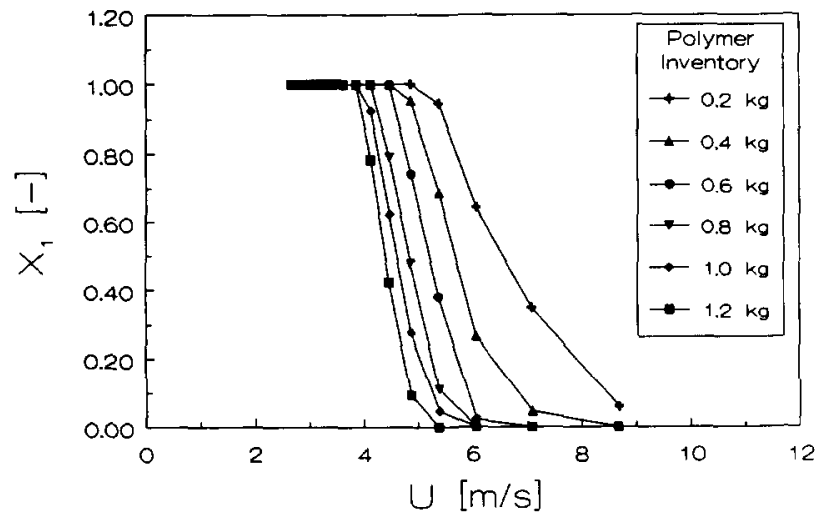

Fig. 8. Experimental values of the mixing parameter index $X_{1}$ [Eq (14)] against the local gas velocity. Particles applied are $1.5 \mathrm{~kg}$ silica $0.0030 \mathrm{~m}$ and a variable amount of polymer $0.0030 \mathrm{~m}$ (Table 1)

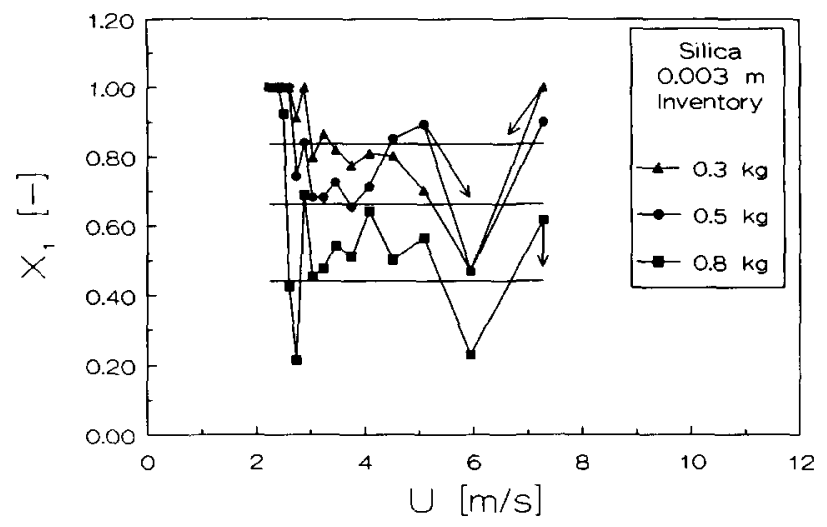

Fig. 9. Experimental values of the mixing parameter $X_{1}$ [Eq. (14)] against the local gas velocity. Particles applied are $1 \mathrm{~kg}$ silica 0.0023 $\mathrm{m}$ and a variable amount of silica $0.0030 \mathrm{~m}$ (Table 1). Horizontal lines: the calculated values for complete mixing $\left(X_{i, \text { ext }}\right)$ for sets of data points denoted by the arrows.

Table 3

Values of the separation tendency $W$ compared to the experimentally observed behaviour in the FGSFB described in the experimental section

\begin{tabular}{ll}
\hline$W$ & Type of system \\
$\left(\mathrm{kg} / \mathrm{m}^{3}\right)$ & \\
\hline$<50$ & Well mixed \\
$50-75$ & Intermediate \\
$>75$ & Completely separated \\
\hline
\end{tabular}

paring data of all systems investigated to the mixing state, it is deduced that a well-mixed situation occurs for $W$ values below 50, while complete separation is found for $W$ above 75 (Table 3). These findings are also supported by the visual observation of intermediate mixing for the glass-polymer system, where the calculated $W$ is also below 50 .

It should be emphasized that these limiting values are dependent on the geometry of the experimental apparatus, on the availability and shape of the internals and on the types of particle employed. Because of this dependency of the limiting values of $W$ on the circumstances encountered, it is tempting to use these as a quantitative measure of the mixing action in different fluidized beds.

\subsection{Design procedure}

A practical procedure is proposed to calculate the bed composition of binary mixed or separated beds. First, the required volume of particles for the process under consideration must be identified (conversion, adsorption). Furthermore, the most beneficial type of mixing (i.e. completely mixed or separated) for a particular process should be established. If the particle properties are known, the porosity profile [Eqs. (4)-(6) and (17)] and the pressure-drop profile [Eq. (14)] can 
be calculated for beds of the separate single-particle type FGSFBs. From the values thus obtained, the separation tendency $W$ can be obtained using Eq. (19). Subsequently it can be deduced whether a systems tends to be completely separated or well mixed or whether it behaves intermediately. It may be required to alter the properties of the particles to obtain the desired degree of mixing.

If, according to the above procedure, a completely separated bed is to be expected with the flotsam bed directly on top of the jetsam, the axial position of the sudden change in $\mathrm{d} P / \mathrm{d} H$, indicating the top of the jetsam bed, can be calculated from the required particle volume of these particles. In a completely separated FGSFB, the reactor volume occupied by jetsam particles is calculated by:

$$
V_{2}=\int_{H_{\mathrm{ent}}}^{H_{\mathrm{tog}, 2}}\left(1-\epsilon_{2}\right) A_{\perp}(H) \mathrm{d} H
$$

which yields $H_{\mathrm{top}, 2}$ if $V_{2}=V_{2 \text {, req. }}$. The volume occupied by the flotsam particles is calculated in the same way:

$$
V_{1}=\int_{H \text { top, } 2}^{H_{\text {top, }}}\left(1-\epsilon_{1}\right) A_{\perp}(H) \mathrm{d} H
$$

For a completely mixed bed, $X_{1}$ can be derived from the relative volumes of particles added and Eq. (11). This value is now assumed to be independent of the height $H$. The height of the bed $\left(H_{\text {top }}\right)$ follows from the required volume of flotsam particles $V_{\mathrm{tot}, 1}$ [Eq. (22)] or that of jetsam particles, $V_{\text {tot, } 2}$ [Eq. (23)]:

$V_{\mathrm{tot}, 1}=X_{1} \int_{0}^{H_{\mathrm{top}}}\left(1-\epsilon_{1}^{\mathrm{o}}\right) A_{\perp}(H) \mathrm{d} H$

$V_{\text {tot, } 2}=\left(1-X_{1}\right) \int_{0}^{H_{\text {top }}}\left(1-\epsilon_{2}^{\mathrm{o}}\right) A_{\perp}(H) \mathrm{d} H$

in combination with Eqs. (4)-(6) and (17) for calculation of the height-dependent porosity $\epsilon$. With these equations, the column volume required can be calculated using:

$$
V_{\mathrm{tot}}=V_{\mathrm{tot}, 1}+V_{\mathrm{tot}, 2}
$$

Furthermore, the pressure drop due to the presence of particles in both beds can be determined by:

$$
\begin{aligned}
\mathrm{d} P_{\text {tot }}= & \int_{H_{\text {ent }}}^{H_{\text {top }}}\left[1,\left(1-\epsilon_{1}\right)\left(\rho_{\mathrm{s}, 1}-\rho_{\mathrm{g}}\right) g\right. \\
& \left.+\left(1-X_{1}\right)\left(1-\epsilon_{2}\right)\left(\rho_{\mathrm{s}, 2}-\rho_{\mathrm{g}}\right) g\right] \mathrm{d} H
\end{aligned}
$$

where the appropriate value of $X_{1}$ should be included, i.e. zero for $0-H_{2}$ and 1 for $H_{2}-H_{1}$ for completely separated beds and $X_{1, \text { ext }}$ [Eq. (11)] for completely mixed beds.

\section{Conclusions}

The floating gas-solids fluidized bed has been applied as a binary fluidized bed and it was shown that its appearance may vary from well-mixed to completely separated. Visual observation showed that intermediate mixing regimes could exist, as well as the extreme situations of complete separation and good mixing of particles. These observations were supported by the interpretation of measured pressure-drop profiles due to the presence of particles: different degrees of mixing were reported, varying from completely separated to intermediately and well-mixed.

To explain the results of visual observations and measurements, a model is formulated quantifying the separation tendency $W$ in a binary fluidized bed. $W$ is derived from a force balance for a particle present in a binary fluidized bed. The starting point is the assumption that a particle is surrounded by the same amount of fluid as in a single-particle type fluidized bed at the same conditions. Both the particle and the fluid are successively treated as a cell or 'pseudoparticle'. The separation tendency $W$ is defined as the difference in density of such cells containing different particle types at the same conditions.

Observed mixing states of the FGSFB have been correlated to the value of the separation tendency, to yield the limits of $W$ for completely separated and for completely mixed beds. Complete mixing was observed if the separation tendency $W$ was smaller than 50 , while complete separation was found for values higher than 75. Modelling the binary FGSFB by applying the concept of unit cells seems to be a promising approach: even the behaviour of the glass-polymer system [a binary bed of small $(0.8 \mathrm{~mm})$ but dense $\left(2900 \mathrm{~kg} / \mathrm{m}^{3}\right)$ glass particles and large $(3.0 \mathrm{~mm})$ but less dense $(1400 \mathrm{~kg} /$ $\mathrm{m}^{3}$ ) polymer particles] in a FGSFB was well predicted.

The above-mentioned limiting values for $W$ are expected to be system-dependent: they can be influenced by the column geometry, by the presence and shape of grids and hopefully to a small extent by the properties of the particle types employed. In that case it could be applied as a measure for comparing the mixing action in different (types of) fluidized beds. Many more experiments are required to establish the general applicability of the concept of the separation tendency $W$ for other fluidized systems.

A design procedure for either completely mixed or separated beds is proposed requiring the system geometry, the properties of the particle types used, the limiting values of the separation tendency, the porosity-velocity relation in the single-particle type 
FGSFBs [2] (same conditions) and the respective amounts of both particle types employed.

\section{List of symbols}

$\begin{array}{ll}A & \text { area }\left(\mathrm{m}^{2}\right) \\ C & \text { friction factor [Eq. (4)] }(-) \\ d & \text { diameter (m) } \\ D & \text { column diameter at the column entrance }(\mathrm{m}) \\ f & \text { force per unit volume }\left(\mathrm{N} / \mathrm{m}^{3}\right) \\ F & \text { porosity function }(-) \\ g & \text { specific gravity, } 9.81\left(\mathrm{~m} / \mathrm{s}^{2}\right) \\ H & \text { axial coordinate }(\mathrm{m}) \\ n & \text { exponent in the Richardson and Zaki equation } \\ & \text { [Eq. (1)] }(-) \\ P & \text { number of particles }(-) \\ P & \text { pressure }\left(\mathrm{N} / \mathrm{m}^{2}\right) \\ \mathrm{Re} & \text { Reynolds number }(-) \\ U & \text { superficial fluid velocity }(\mathrm{m} / \mathrm{s}) \\ V & \text { volume }\left(\mathrm{m}^{3}\right) \\ W & \text { unit cell density difference defined in Eq. (10) } \\ & \text { (separation tendency) }\left(\mathrm{kg} / \mathrm{m}^{3}\right) \\ X & \text { volume fraction of unit cells defined in Eq. }(9) \\ & (-)\end{array}$

\section{Greek letters}

$\begin{array}{ll}\alpha & \text { apex angle }\left({ }^{\circ}\right) \\ \epsilon & \text { porosity }(-) \\ \rho & \text { density }\left(\mathrm{kg} / \mathrm{m}^{3}\right) \\ \Phi & \text { flowrate }\left(\mathrm{m}^{3} / \mathrm{h} \text { or } \mathrm{m}^{3} / \mathrm{s}\right)\end{array}$

Sub- and superscripts

$\begin{array}{ll}\text { b } & \text { buoyant } \\ \text { d } & \text { drag } \\ \text { ext } & \text { mixed conditions } \\ \text { f } & \text { fluid } \\ \text { fr } & \text { friction } \\ \text { i } & \text { particle type (either } 1 \text { or } 2 \text {, flotsam or jetsam) } \\ \text { net } & \text { net value } \\ \text { o } & \text { value in single-particle type bed } \\ \text { p } & \text { particle } \\ \text { req } & \text { required value } \\ \text { sus } & \text { suspension } \\ \text { t } & \text { terminal conditions } \\ \text { top } & \text { top of bed } \\ \text { tot } & \text { total } \\ \text { uc } & \text { unit cell } \\ 1 & \text { flotsam } \\ 2 & \text { jetsam } \\ \perp & \text { perpendicular } \\ * & \text { specificed defined value [see Eq. (4)] }\end{array}$

\section{Acknowledgement}

The authors gratefully acknowledge the financial and experimental support provided by KEMA NV, Arnhem, The Netherlands.

\section{References}

[1] G. Kwant, W. Prins, W.P.M. van Swaaij, P. Lodder and J.B. Lefers, in O.E. Potter and D.J. Nicklin (eds.), Fluidization VII, Proc. Seventh Engineering Foundation Conf. Fluidization, Brisbane, Australia, 1992, Engineering Foundation, New York, USA, 1992, p. 667.

[2] G. Kwant, Ph.D. Thesis, Twente University of Technology, Enschede, The Netherlands, 1994.

[3] A.K. Hjalmarsson, IEA Coal Research, IEA Coal Research, London, 1990.

[4] J.J.P. Biermann, Ph.D. Thesis, Twente University of Technology, The Netherlands, 1989.

[5] P.N. Rowe and A.W. Nienow, Powder. Technol., 15 (1976) 141

[6] A.W. Nienow and T. Chiba, in J.R. Davidson, R. Clift and D. Harisson (eds.), Fluidization, Academic Press, London, 1985.

[7] T. Maruyama, H. Maeda and T. Mizushina, J. Chem. Eng. Jpn., 17 (1984) 132.

[8] J.F. Richardson and W.N. Zaki, Trans. Inst. Chem. Eng., 32 (1954) 35.

[9] M.J. Lockett and H.M. Al-Habbooby, Trans. Inst. Chem. Eng., 51 (1973) 281.

[10] Z. Zhang and J.M. Beeckmans, Can. J. Chem. Eng., 68 (1990) 932 .

[11] J.M. Beeckmans and Z. Yu, in O.E. Potter and D.J. Nicklin (eds.), Proc. Seventh Foundation Conf. Fluidization, Brisbane, Australia, 1992, Engineering Foundation, New York, USA, 1992, p. 643 .

[12] C.E. Capes and A.E. Mcllhinney, AIChE J., 14 (1968) 917.

[13] J.R. Grace and D. Harrison, Chem. Proc. Eng., (1970) 127.

[14] G. Donsi, G. Ferrari and B. Formisani, Can. J. Chem. Eng., 67 (1989) 185.

[15] R. Samson, C.E. Brakel, A.M. Scott and K. Chandrasekharan, Chem. Eng. Sci., 43 (1988) 2215.

[16] P.N. Rowe and K.S. Sutherland, Trans. Inst. Chem. Eng., 42 (1964) T55.

[17] M. Hemati, K. Spieker, C. Laguerie, R. Avvarez and F.A. Riera, Can. J. Chem. Eng., 68 (1990) 768.

[18] J.P. Sutherland, G. Vassilatos, H. Kubota and G.L. Osberg, AIChE J., 9 (1963) 437.

[19] J. van Deemter, in J.R. Davidson, R. Clift and D. Harisson (eds.), Fluidization, Academic Press, London, 1985.

[20] G.H. Webster and J.J. Perona, AIChE J., 34 (1988) 1398.

[21] J.H. Masliyah, Chem. Eng. Sci, 34 (1979) 1166.

[22] S. Mirza and J.F. Richardson, Chem. Eng. Sci., 34 (1979) 447

[23] A.K.A. Juma and J.F. Richardson, Chem. Eng. Sci., 38 (1983) 955.

[24] V.S. Patwardhan and C. Tien, Chem. Eng. Sci., 40 (1985) 1051

[25] H.S. Law, J.H. Masliyah, R.S. MacTaggert and K. Nandakumar, Chem. Eng. Sci., 42 (1987) 1527.

[26] M.A. Al-Naafa and M.S. Selim, Can. J. Chem. Eng., 67 (1989) 253.

[27] M.A. Selim, A.C. Kothari and R.M. Turia, AIChE Symp. Ser. Fluidization and Fluid Particle Systems, 79 (1983a) 103-109.

[28] M.S. Selim, A.C. Kothari and R.M. Turia, AIChE J., 29 (1983b) 1029-1038.

[29] E. Bamea and J. Mizrahi, Chem. Eng. J., 5 (1973) 171. 
[30] P.U. Foscolo, L.G. Gibilaro and S.P. Waldram, Chem. Eng. Sci., 38 (1983) 1251.

[31] R. Di Felice, P.U. Foscolo, L.G. Gibilaro and S. Rapagna, Chem. Eng. Sci., 46 (1991) 1873.

[32] J. Garside and M.R. Al-Dibouni, Ind. Chem. Proc. Des. Dev., 16 (1977) 206.

[33] K. Ridgeway and H.K. Sim, Chem. Proc. Eng., (1966) 281.
[34] R.K. Saksena and C.R. Mitra, Period. Polytechn. Chem. Eng., 17 (1973) 289.

[35] R.P. Levey, A. de la Garza, S.C. Jacobs, H.M. Heidt and P.E. Trent, Chem. Eng. Prog., 56 (1960) 43.

[36] B.S. Malone, Ph.D. Thesis, University of London, UK, Battersea College of Technology, 1963.

[37] K.S. Sutherland, Trans. Inst. Chem. Eng., 39 (1961) 188. 